\title{
Editorial
}

\section{Comprehensive Perinatal Centers in the Era of Managed Care}

\section{John V. Hartline, MD}

Included in this issue of the Journal is an excellent description of 20 years' experience of the New Hampshire Perinatal Program.

The concept that the perinatal center had responsibilities beyond the development of excellence in care of the referred patient was implemented in programs under the guidance of Dr. Stanley Graven in Wisconsin, Dr. Joseph Butterfield in Colorado, and others in the late 1960s and early 1970s. Components of the perinatal center, including perinatal outreach, were originally described in Toward Improving the Outcome of Pregnancy, published by the March of Dimes in 1976. At a time when neonatologists were few and when they could focus their practice, teaching, and research in larger perinatal centers with large volumes of referred patients, many participated in perinatal outreach programs with the realization that perinatal care is an essential component of community medicine. A relationship between community medicine and regional perinatal centers could improve the outcome of pregnancy not only in perinatal centers but in community hospitals as well. Additionally, for those patients requiring the highest levels of intensive care, both quality outcome and cost efficiency argued in favor of the regionalized perinatal center.

As the number of neonatologists increased beyond the needs of large perinatal centers, many small to medium sized hospitals hired neonatologists for care of mostly inborn infants. This has led to deregionalization and to the development of numerous small intensive care units which, in some studies, have not been able to document the same degree of quality outcome as experienced in larger centers. Additionally, as managed care has taken over health financing in many areas of the country, constituent parts of perinatal center activities have been analyzed for their degree of revenue production. The perinatal outreach education program was one of the more vulnerable components of the perinatal center in that it generated little, if any, revenue and required considerable time and expertise from perinatal center personnel. The New Hampshire Perinatal Program clearly documents the phenomenal amount of effort and expertise required for a high-quality perinatal outreach education program. Unfortunately, managed care has had little interest in contributing to

Bronson Methodist Hospital, Kalamazoo, MI

Address 1 correspondence and reprint requests to John V. Hartline, MD, Bronson Methodist Hospital, Kalamazoo, MI 49007. such activities and in many ways has supported the cost-inefficient, quality-inefficient process of deregionalization. This has been driven in part by the lack of opportunities at larger perinatal centers for professional practice, teaching, or research for many fully trained neonatologists.

As neonatologists have proliferated, the participation of general pediatricians in the care of the sick newborn has diminished rapidly. This has led to a reduction in time in the newborn intensive care unit for pediatricians-in-training, leaving the graduating pediatric resident with fewer and fewer skills in care of the high-risk newborn. In some situations, loss of the pediatric residents and/or neonatal fellows in the newborn intensive care unit have resulted in a staffing crisismeaning that the faculty or attending neonatologists have to come in and participate in hands-on neonatal intensive care!

In response to this, we see many looking for relief from pediatric or neonatal nurse practitioners, partially trained neonatal clinicians, or physician assistants. At the same time, many of the graduates from the fellowship programs of these very same hospitals are unable to find a professional environment allowing them to reach fulfillment in the type of practice for which they were trained at the perinatal center.

The New Hampshire Perinatal Program demonstrates many diverse activities of the busy perinatal center in which the fully trained neonatologist could find professional satisfaction and apply the many analytic, clinical, and teaching skills acquired during the neonatal fellowship program. It is time for the perinatal centers who have trained follows in the past and who are feeling this staffing pinch to reach out to those neonatologists who would like to return to the busy perinatal center and participate in clinical care, teaching, perinatal outreach, and clinical evaluation. This would then allow the academic neonatologists to rededicate their time for the absolutely necessary research requirements of our specialty.

Regionalized perinatal care goes beyond the care of the complicated pregnancy and the sick newborn. As described in the New Hampshire Perinatal Program, regionalized perinatal care is as much about prevention as it is about care of the sick. Regionalization of newborn intensive care and perinatal care has been a major factor in the substantial reduction in perinatal mortality over the past 30 years. However, the increase in survival has not been only the result of improved care of the sick, but it has also been a result of the interactions between many perinatal centers with the hospitals, physicians, and nurses whom they serve. Managed care needs to understand that it can not hope for continued improved outcome in perinatal care without a commitment to the full spectrum of activities of perinatal cen- 
ters as described in the New Hampshire Perinatal Program. A full service perinatal center must have a program of perinatal outreachcommitted to improving the level of perinatal care at each birthing venue within its served area. Additionally, managed care must under- stand that efficient and high-quality intensive care can be achieved only through the centralization or regionalization of those patients who are benefited by the combined, cooperative, multidisciplinary experience available at the regional perinatal center. 